\title{
Possible axonal regrowth in late recovery from the minimally conscious state
}

\author{
Henning U. Voss, ${ }^{1}$ Aziz M. Uluğ, ${ }^{2}$ Jonathan P. Dyke, ${ }^{1}$ Richard Watts, ${ }^{3}$ \\ Erik J. Kobylarz, ${ }^{4}$ Bruce D. McCandliss, ${ }^{5}$ Linda A. Heier, ${ }^{2}$ Bradley J. Beattie, 6 \\ Klaus A. Hamacher, ${ }^{1}$ Shankar Vallabhajosula, ${ }^{1}$ Stanley J. Goldsmith, ${ }^{1}$ \\ Douglas Ballon, ${ }^{1}$ Joseph T. Giacino, ${ }^{7}$ and Nicholas D. Schiff ${ }^{4}$
}

\begin{abstract}
${ }^{1}$ Citigroup Biomedical Imaging Center and 2Department of Radiology, Weill Medical College of Cornell University, New York, New York, USA. ${ }^{3}$ Department of Physics and Astronomy, University of Canterbury, Christchurch, New Zealand. ${ }^{4}$ Department of Neurology and Neuroscience and ${ }^{5}$ Sackler Institute for Developmental Psychobiology, Weill Medical College of Cornell University, New York, New York, USA. ${ }^{\circ}$ NeuroOncology Laboratory, Memorial Sloan-Kettering Cancer Center, New York, New York, USA. JFK Johnson Rehabilitation Institute, Edison, New Jersey, USA.
\end{abstract}

\begin{abstract}
We used diffusion tensor imaging (DTI) to study 2 patients with traumatic brain injury. The first patient recovered reliable expressive language after 19 years in a minimally conscious state (MCS); the second had remained in MCS for 6 years. Comparison of white matter integrity in the patients and 20 normal subjects using histograms of apparent diffusion constants and diffusion anisotropy identified widespread altered diffusivity and decreased anisotropy in the damaged white matter. These findings remained unchanged over an 18-month interval between 2 studies in the first patient. In addition, in this patient, we identified large, bilateral regions of posterior white matter with significantly increased anisotropy that reduced over 18 months. In contrast, notable increases in anisotropy within the midline cerebellar white matter in the second study correlated with marked clinical improvements in motor functions. This finding was further correlated with an increase in resting metabolism measured by PET in this subregion. Aberrant white matter structures were evident in the second patient's DTI images but were not clinically correlated. We propose that axonal regrowth may underlie these findings and provide a biological mechanism for late recovery. Our results are discussed in the context of recent experimental studies that support this inference.
\end{abstract}

\section{Introduction}

The minimally conscious state (MCS) (1) denotes a specific level of functional recovery of severely brain-injured patients who demonstrate unequivocal, but intermittent, behavioral evidence of awareness of self or their environment. This condition potentially produces a unique burden of isolation, motivating efforts to better understand brain function in MCS patients in the hope of promoting further recovery (2). Here we report magnetic resonance diffusion tensor imaging (DTI) findings from 2 patients following severe traumatic brain injuries. Our index patient recovered reliable communication after remaining in MCS for 19 years whereas the second patient remained in MCS at the time of study 6 years after injury.

DTI (refs. 3, 4 and references therein) is a novel imaging modality capable of characterizing specific white matter pathologies such as atrophy and diffuse axonal injury. The preferential diffusion direction of water in myelinated white matter locally coincides with the direction of white matter fiber bundles in the brain, and the diffusion constant and anisotropy depend on microscopic tissue properties such as density, diameter, and geometry of myelinated fibers. These properties allow DTI to characterize white matter damage and possible neuronal reorganization in the setting of brain injuries. In this study, DTI was used to evaluate white matter integrity across the cerebrum in both patients and in the first patient to characterize changes in intracortical and cerebellar white matter connectivity patterns across 2 studies 18 months apart. We

Nonstandard abbreviations used: CSF, cerebrospinal fluid; DTI, diffusion tensor imaging; FA, fractional anisotropy; FDG, fluorine-18 fluorodeoxyglucose; MCS, minimally conscious state; MPO, medial parietal, occipital; ROI, region of interest.

Conflict of interest: The authors have declared that no conflict of interest exists.

Citation for this article: J. Clin. Invest. 116:2005-2011 (2006). doi:10.1172/JCI27021. compare our DTI findings in the first patient with fluorine-18 fluorodeoxyglucose-PET (FDG-PET) measurements of the local metabolic rate of brain tissue. The results of these complementary modalities converge, providing evidence of reorganization of the patient's white matter, possibly facilitating functional recovery.

Case report patient 1 . The patient is a 39 -year-old male who at age 19 suffered a severe closed head injury in a motor-vehicle accident. Remarkably, this patient spontaneously emerged from MCS 19 years following the initial injury and recovered spoken language. He initially remained in a coma for 1 to 2 weeks followed by further recovery to a vegetative state and subsequently a level of function consistent with MCS within several months of injury. Although gradual improvements in responsiveness were noted over an ensuing 19-year period, the patient was unable to communicate using gesture or verbal output. Limited head nodding and grunting were only inconsistently present. Eight months prior to our first evaluation, he spoke his first word after his brain injury, "mom," which was followed by a recovery over a period of several days of increasingly fluent, but dysarthric, speech and reliable communication.

At the time of the first DTI evaluation, motor examination revealed bilateral upper extremity contractures with active movement noted on the left side only. There was increased extensor tone bilaterally in the lower extremities accompanied by intermittent clonus, bilateral triple flexion responses, and extensor plantar reflexes. Oculomotor examination showed near-continuous random eye movement (with full range of movement). Saccades and pursuits were intact. Motor speech was fluent but severely dysarthric due in part to dystonic tongue movements. Language output was characterized by a pronounced logorrheic tendency. He was oriented to person but did not know his age, selecting "less than 20 years old" when provided with a 2-alternative, 

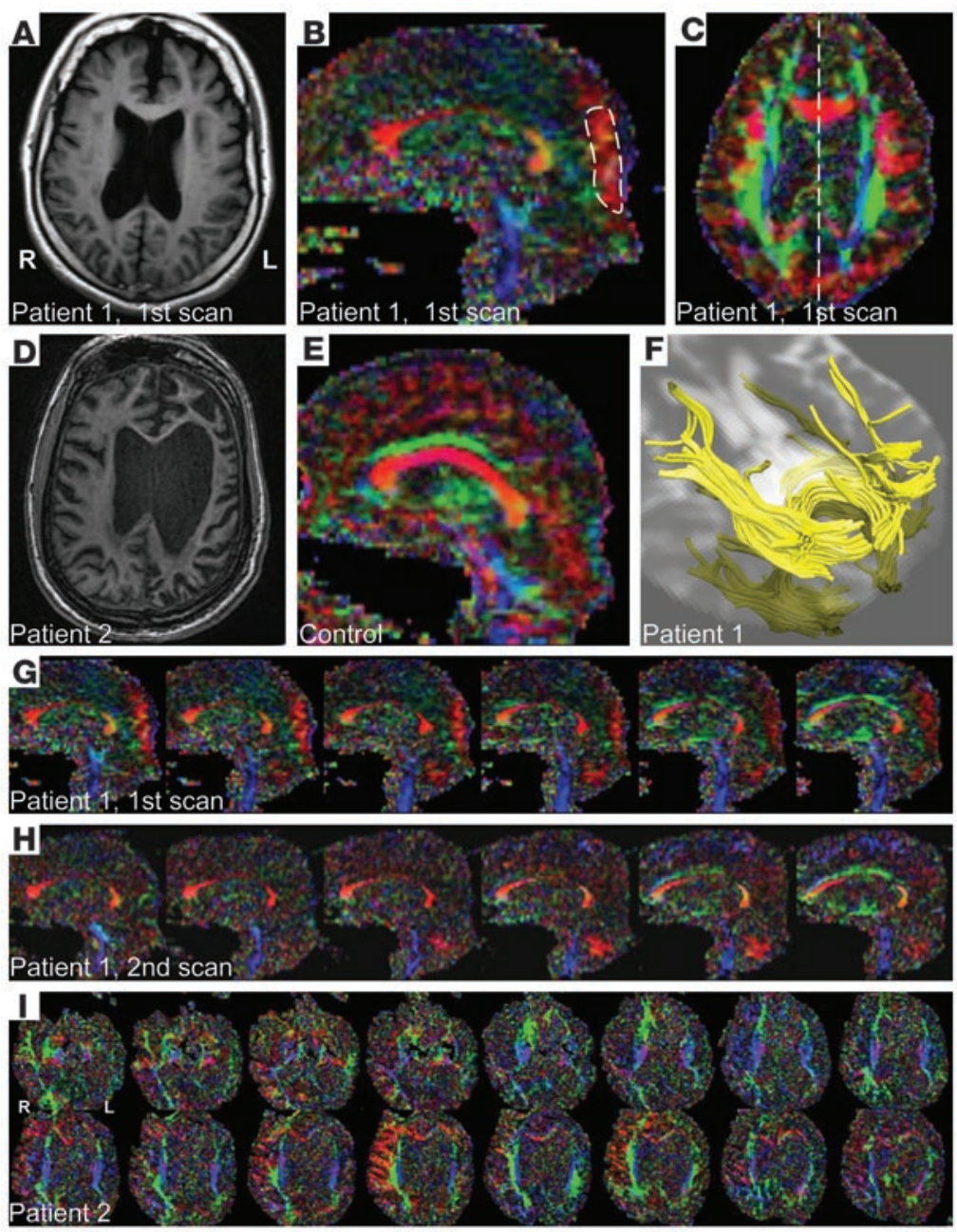

\section{Figure 1}

MRI data: structural images, FA maps, and fiber reconstruction. (A-E) Axial T1-weighted anatomical images (A, patient 1; D, patient 2), sagittal FA color maps (B, patient 1; E, normal control), and axial FA color map of patient 1 (C) corresponding to slice in $\mathbf{A}$. Color is composed of red, blue, and green, indexing whether anisotropy is most pronounced from left to right, superior to inferior, or anterior to posterior, respectively. The patient's MPO ROI is marked by a dashed line in B, and the slice position of $\mathbf{B}$ corresponds to the dashed line in C. The normal control subject with the most pronounced anisotropy in the MPO region is identified as a square mark in Figure 2J. (F) White matter fiber tracts of the patient in the MPO region of the brain, overlaid onto an axial T2-weighted image. (G) Sequence of parasagittal FA maps of patient 1, first scan. The images are ordered from left to right and the space between them is $1.7 \mathrm{~mm}$. (H) Sequence of parasagittal FA maps for patient 1 obtained from the second scan, with the same spacing as before. In the second scan, the vermis appears more and the MPO region less pronounced. (I) Axial FA maps of patient 2. The images are ordered from inferior to superior and have a spacing of $2.5 \mathrm{~mm}$. forced-choice response format. He misidentified his location and denied that he had been transported to his current location by air 48 hours earlier. He indicated that he did not know the current year and selected "1984" (the year he was injured) from a list of 4 alternatives. There were no discernible paraphasic or dysnomic errors in conversation and no evidence of alexia on single-word reading tasks. Confrontation naming was accurate across 10 consecutive trials involving high-frequency items. Phonemic (8 items in 60 seconds) and semantic fluency (7 items in 60 seconds) were severely impaired due to rapid loss of set, perseverative errors, and behavioral disinhibition (e.g., he began making animal noises while generating names of animals).

Eighteen months later, at the time of the second DTI scan, several areas of neurological improvement were identified. Reassessment of motor functions demonstrated some functional recovery of the left upper extremity (i.e., he was able to point but could not perform self-care tasks such as using a spoon). $\mathrm{He}$ remained unable to use the right upper extremity. Most notably, both lower extremities, which were paretic on initial evaluation, showed improvements in strength to at least $4+/ 5$ on volitional movement. He was able to extend both lower extremities when flexed at the knee. These movements were poorly controlled and tended to occur in a ballistic fashion. He also demonstrated a new ability to assume a bridging position in which both legs pushed down to elevate the lower back. Cognitively, there was an overall increase in baseline arousal accompanied by generalized improvement in attentional focus and response persistence. He was able to count from 1 to 25 without interruption and completed a 90-second auditory vigilance, detecting 19 of 30 targets with 1 false-positive error. Speech intelligibility improved, and there was a noticeable decrease in the severity of the logorrhea. He remained oriented to person only and again refused to accept that he had traveled by plane from his home 24 hours earlier. Conversational speech remained free of paraphasic and dysnomic errors, there were no errors on confrontation naming $(20 / 20)$, and single-word reading was accurate. Verbal fluency was essentially unchanged (trial 1: 8 items in 60 seconds; trial 2: 4 items). He continued to deny awareness of any physical or cognitive problems, typically providing rational but inaccurate explanations for the problems he encountered when symptoms were elicited on examination.

Case report patient 2 . The patient is a 24 -year-old male who sustained severe traumatic brain injury in a motor-vehicle accident 6 years prior to examination with DTI. He remained in a vegetative state, as judged by repeated examinations for over a year following the injury. Approximately 17 months into the course of the patient's illness, standardized testing identified reliable vertical visual pursuit, clear evidence of object manipulation with the left 
A

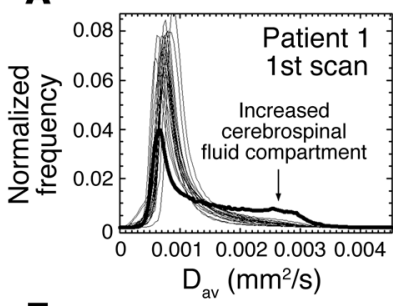

E

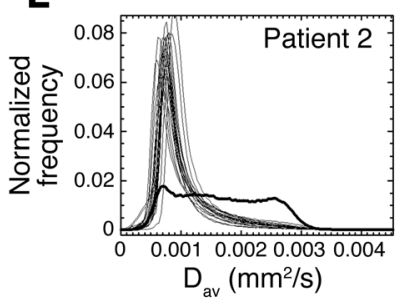

I

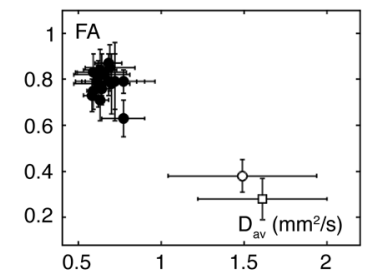

B

F
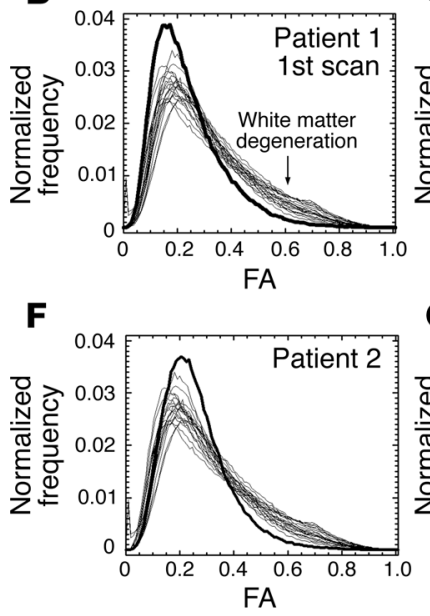

J

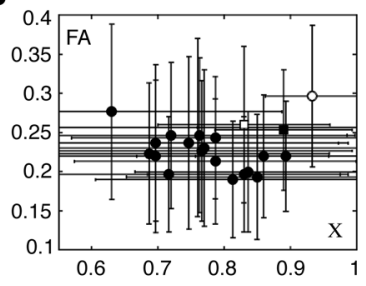

C

G
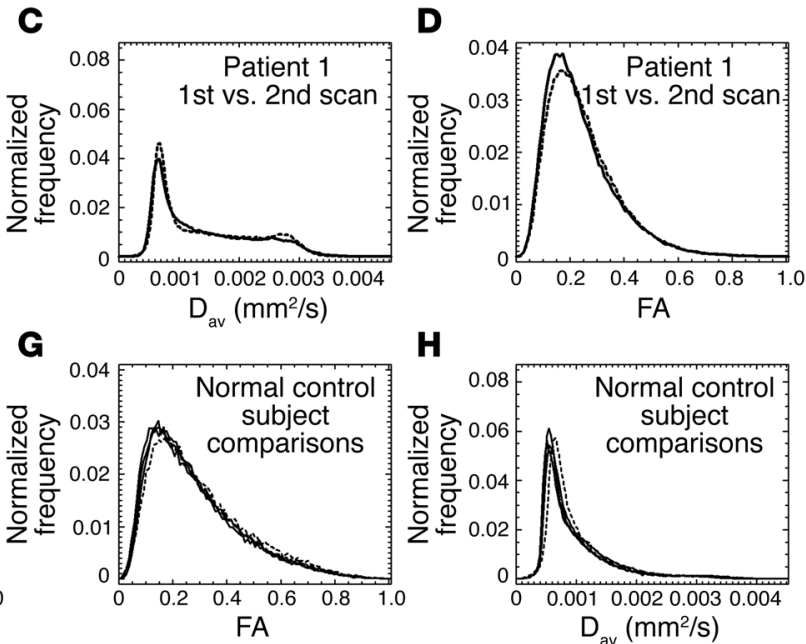

H

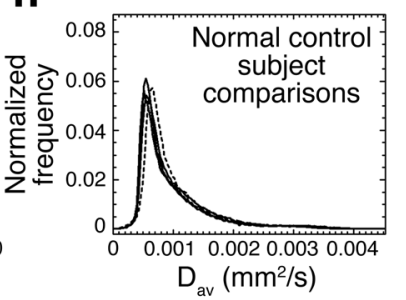

K

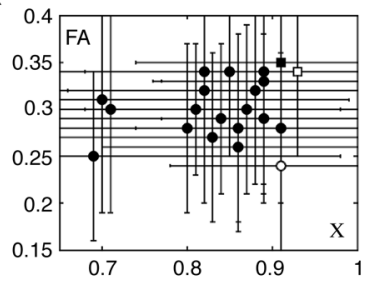

Figure 2

Quantification of white matter reorganization. (A) Histogram of average apparent diffusion constant ( $\mathrm{D}_{\mathrm{av}}$ ) of patient 1 (bold line) and the 20 normal control subjects over all voxels in the brain. The histogram of the patient shows an enlarged compartment of CSF as compared with normal subjects. (B) Same as A for the FA. The patient's histogram lacks large FA values, characteristic for overall white matter degeneration. (C and D) Comparison of histograms of patient 1 for the first (solid lines) and second scan 18 months later (dotted lines). (E and F) Same as A and $\mathbf{B}$ for the second patient. ( $G$ and $\mathbf{H}$ ) Histograms of $D_{a v}$ and FA of a normal control subject who was scanned 3 times within a week (solid lines) and then once 17 months later (dashed lines). (I) FA versus $D_{a v}$ for patient 1 (open circle, first scan; open square, second scan) and the 20 normal control subjects (closed circles) for an ROI in the medial corpus callosum, showing pronounced white matter atrophy in the patient's brain. Bars indicate standard deviations over the ROI. (J) FA- $\chi$ diagram of the MPO region of patient 1 (open circle, first scan; open square, second scan) and 20 normal control subjects (closed circles). $\chi$ quantifies the right-left component of the main diffusion direction whereas FA quantifies the overall anisotropy. The closed square marks the control subject referred to in Figure 1E. Bars indicate mean standard deviations over ROI as measured by 3 of the authors. (K) Same as $\mathbf{J}$ but for the inferior part of the vermis. Bars indicate standard deviations over the ROI.

upper extremity (grasp and release), and occasional episodes of command following using eye gaze. These changes signaled transition from the vegetative state to the MCS. He continues to be quadriparetic with inconsistent evidence of purposeful movement of the left upper extremity only. There have been no observable clinical changes over the last 4 years.

\section{Results}

The anatomical MRI images of both patients show widespread cerebral atrophy (Figure 1, A and D). Notably, the lateral ventricles were increased in size, and the corpus callosum and the periventricular white matter were diminished. Color anisotropy maps demonstrate the predominant white matter fiber direction, with different colors indicating different directions (Figure 1, B, C, and $\mathrm{G}-\mathrm{I}$ ). The intensity of the color is proportional to the diffusion anisotropy. Anisotropy, measured by the fractional anisotropy (FA) index, was large for the hindered diffusion of water, as it is in the presence of myelinated axons, and smaller for more isotropic regions in the brain, such as normal gray matter or cerebrospinal fluid (CSF). The color FA maps of the patients demonstrate a marked reduction of volume and diffusion anisotropy of the medial corpus callosum and other parts of the brain compared with normal control subjects (Figure 1E).

To compare both patients' regional white matter integrity with normal control subjects, we performed a quantitative analysis of the DTI data. Histograms of the average diffusion constant (Figure 2, A and E) and FA (Figure 2, B and F) correlate with global injury to the brain white matter in both patients, showing a marked shift toward lower average diffusion values in white matter (5) and an increased CSF compartment. Overall, diffusion was increased, as expected, whereas anisotropy was decreased. In the aggregate, these findings are consistent with the patients' having both suffered severe diffuse axonal injury (Grade $3 \mathrm{DAI}$ ).

A detailed quantitative analysis of the corpus callosum in patient 1 indicated anisotropy loss across substructures: whereas the FA values for the genu of the corpus callosum were within the normal range (first scan, $F A=0.70 \pm 0.14$; second scan, $\mathrm{FA}=0.68 \pm 0.13$; control, $\mathrm{FA}=0.69-0.85$ ), the splenium of the corpus callosum showed significantly lower FA values compared with normal control subjects (first scan, $F A=0.61 \pm 0.20$; second scan, $\mathrm{FA}=0.58 \pm 0.11$; control, FA > 0.69). Neither measurement showed a significant difference between the first and second scan. 


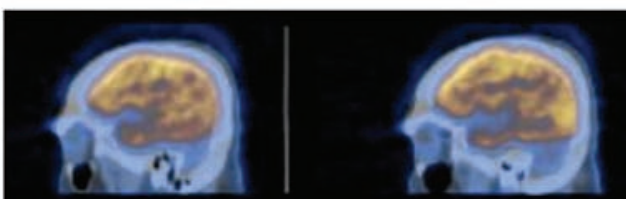

24
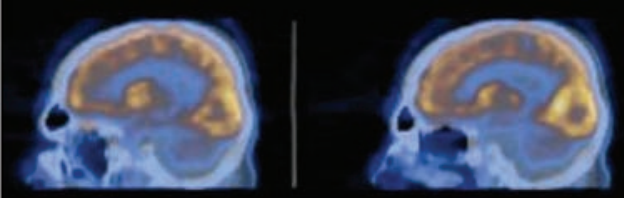

29
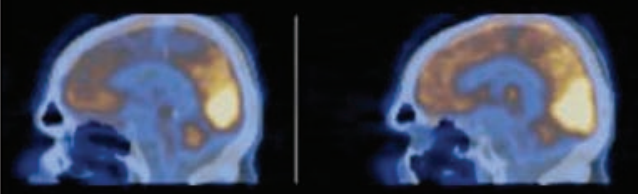

34
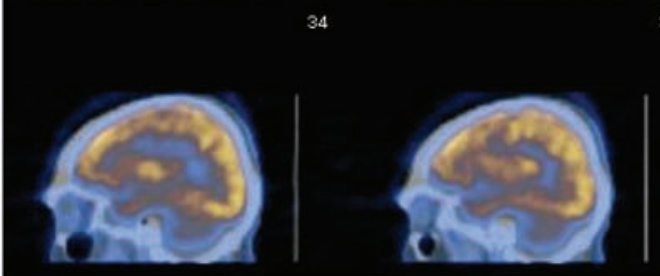

35

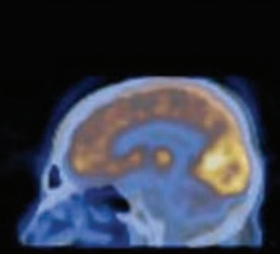

30

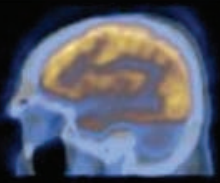

25
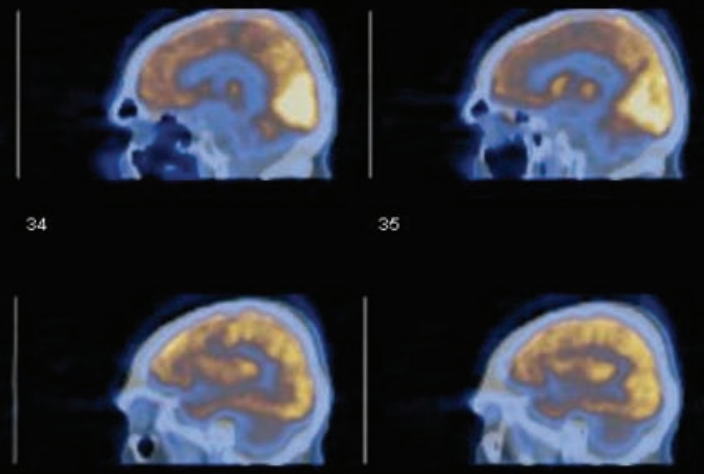

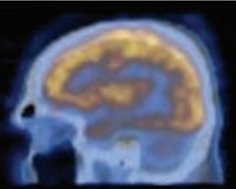

26

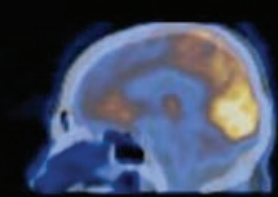

31
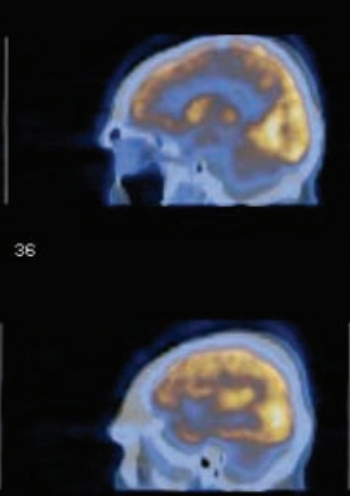

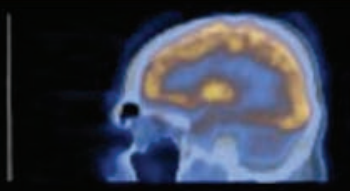

27

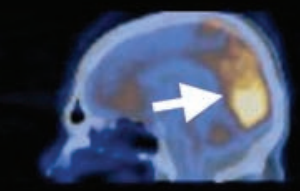

32

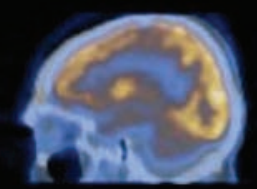

37

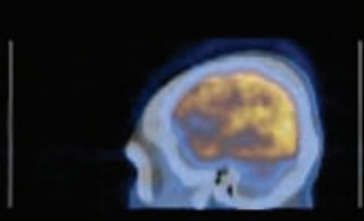

Figure 3

Sagittal PET-CT images of patient 1's brain. The FDG uptake is displayed as brown (smaller uptake value) to white (larger uptake values). The CT image is displayed in bluish tones. The area marked by an arrow corresponds to the area marked in Figure $1 \mathrm{~B}$.

The medial portions of callosum showed marked reduction in anisotropy (first FA $=0.38$; second FA $=0.28$; see Figure $2 \mathrm{I}$ ). This portion of the callosum is very thin, and the measurements were influenced by neighboring tissue with smaller FA (partial volume effect). As an additional and reliable measure for the reproducibility of these values between the 2 scan times of patient 1, we chose the CSF as a large compartment in the brain that could be measured without substantial partial volume effects. For CSF, FA values were normal and equal between the first and second scan (first scan, $\mathrm{FA}=0.17 \pm 0.07$; second scan, $\mathrm{FA}=0.18 \pm 0.04$; control range, $\mathrm{FA}=0.15-0.28$ ). The constancy of CSF values supports the quantitative reproducibility of the 2 scans of patient 1 . Furthermore, Figure 2, C, D, G, and H, shows a comparison of diffusion and FA histograms, respectively, for patient 1 and a normal control subject scanned twice in a period of 17 months. Whereas small fluctuations in the voxel counts were unavoidable, for example due to differing partial volume effects between scans, the difference in histograms is marginal compared with the difference between the patient and the control subject's histograms as shown in Figure 2, A, B, E, and F.

Contrary to the general findings of decreased anisotropy across brain structures in the first scan of patient 1 , there were well-confined regions of pronounced right-left anisotropy in the medial parietal, occipital (MPO) areas of the brain, bilateral to the interhemispheric fissure (visible as the red occipital areas in Figure 1, B and C, and through the sequence of parasagit- tal images in Figure 1G). These fibers were contiguous with the splenium of the corpus callosum (Figure 1F). Based on these qualitative observations, we sought a quantitative comparison of this region in the patient and the normal subjects. As a proxy for the strength of occipital right-left connections, we used 2 measures: the FA and the fiber right-left directionality index, $\chi$, defined as the right-left component of the principal diffusion direction. Whereas FA was assumed to reflect myelinated fiber density, diameters, and microscopic structures such as crossing and branching fibers, we used the right-left directionality index $\chi$ to measure the degree of right-left directionality of fibers, independent of fiber density and diameters.

In the first scan of patient 1 , the MPO areas were significantly larger than in normal controls as is evident from a comparison of FA values $(P=0.05)$ and $\chi(P=0.05)$ with normal controls (Figure $2 \mathrm{~J}$ ). In the second scan, 18 months later, the FA values reduced and were no longer separated from the control group. However, in the second scan, another striking region of right-left anisotropy had become evident in the inferior part of the cerebellar vermis (Figure $1 \mathrm{H}$ ). The increase of FA in the vermis between the 2 scans is statistically highly significant $(P<0.0001$; see Figure $2 \mathrm{~K}$ ).

The marked increase in anisotropy of the cerebellar vermis had a direct clinical correlate in the patient's regaining of limited use of the lower extremities over the 18-month interval between evaluations and improved motor function of the left upper extremity. In addition, although less easily graded on a clinical 
exam, the patient's dysarthric speech pattern notably improved over the same time interval and likely reflected changes in cerebellar function as well.

FDG-PET images of resting metabolism in an awake, eyes-open state provided converging evidence for relatively increased functionality in the MPO area of the brain of patient 1 (Figure 3). Compared with all other brain areas, the MPO regions exhibited the highest resting FDG uptake, both at the time of the first PET scan and in a repeat study 18 months later (not shown). Calculation of standard uptake values based on body weight at the first scan showed that the MPO regions maintained slightly above-normal resting values compared with an approximately $25-30 \%$ reduction from normal values seen across all other brain structures, with the exception of the cerebellar hemispheres, which showed approximately 50\% reductions from normal values, consistent with the patient's quadriparesis. Comparison of the second and first FDGPET scans revealed an approximately $20 \%$ increase in standard uptake values across brain structures without marked qualitative differences. The midline cerebellum standard uptake values, however, increased 50\% in the second PET scan. This independent measurement suggests that the observed DTI changes are related to activity-dependent changes in this part of the structure.

The second patient studied exhibited several unusual features in FA color maps that are suggestive of changes in white matter connectivity. In Figure 1I, axial slices of the second patient's brain are displayed. The less damaged right hemisphere (compare with Figure 1D) in this patient contained several novel-appearing structures, namely thick anterior-posterior connections (green) and strong right-left connections (red) throughout the right frontoparietal cortex that were continuous with the anterior corpus callosum. This asymmetric feature appeared to also receive connections from the thick anterior-posterior fiber tracks. However, as previous studies are not available, we cannot rule out the possibility that these connectivity patterns may have existed prior to injury.

\section{Discussion}

The large changes in anisotropy in patient 1's brain, which otherwise has overwhelming damage consistent with diffuse axonal injury, raise questions about their origin. In the first DTI study of the patient's brain, the bilateral MPO regions had higher-thannormal anisotropy (FA) and directionality $(\chi)$. Their reduction in anisotropy 18 months later suggests that the directionality may have been a transitional stage of an ongoing process. The stability of other strongly directional features of the DTI images and the newly appearing increases in the midline cerebellar anisotropy further support this view.

How can these findings be explained? It is known that a cortical injury creates a particularly favorable environment for sprouting to occur in the intact remaining cortex $(6,7)$. Dancause et al. (8) recently provided anatomical and neurophysiological evidence of extensive axonal regrowth in adult squirrel monkeys, arising months after injury. In their studies, M1 axons in the ventral premotor cortex sought new targets in surviving, intact tissue, with a focused fiber direction orientation. This widespread corticocortical sprouting extended to intact cortical areas distant from the injury, which underwent major neuroanatomical reorganization. During the process of axonal growth, it was further observed that the orientation of the new fibers in the injured animals was significantly more focused than the relatively diffuse orientation of fibers in normal control cases.
The axonal regrowth was correlated with functional motor recovery. A recent human DTI study, also using FA for quantification, showed a complementary finding that white matter plasticity can be observed in adult humans as a result of intensive piano practicing, with most pronounced changes observed in association (corticocortical) fiber connectivity (9). We conjecture that the initially observed increments of FA and $\chi$ may reflect such a distant reorganization since the posterior cortical areas were among the least affected by the patient's brain injury. The normalization of FA and $\chi 18$ months later could be a result of ongoing sprouting of axons towards other targets. Decreasing FA values have been observed during the development of preterm human cerebral cortex (10) and can likely be explained by innervation by corticocortical axons and synaptogenesis, among other processes (11). Similar to the posterior cortical areas, the increasingly strong anisotropy in the cerebellar vermis may reflect an additional location of remodeling; it is notable that in the first scan the directionality index $\chi$ was already large for this region compared with the control group.

There is other evidence for large-scale cortical rewiring in adult monkeys after amputations of digits or limbs, or retinal lesions, which could only be explained by axonal growth (ref. 12 and references therein). Some studies have suggested that axonal regrowth beyond $10 \mathrm{~mm}$ may also be possible and is likely activity dependent (13). To date, such axonal remodeling has only been observed in response to injuries that persisted months to years. Interestingly, late remyelination specifically within the posterior occipital cortices as suggested here in our patient has been observed in studies of normal, aging nonhuman primates (14). Collectively, in the context of current literature, these findings suggest that regrowth and rerouting of the white matter tracts may account for the measured changes in brain structure.

This interpretation is further supported by the observation of strongly preserved metabolic activity in the MPO regions compared across other cerebral structures. In other studies, we have compared metabolic rates of severely brain-injured patients to normal values using the same PET scanning technologies and correlated the findings with other imaging modalities $(15,16)$. A general conclusion of these studies is that regional preservation of the FDG-PET signal correlates with both preserved clinical identifiable functionality and electrophysiological activity. Thus, we interpret the findings of a relatively increased metabolism in the same regions where anisotropy is increased as converging evidence for late reorganization of white matter connections in this patient's MPO regions of the brain.

Even if the initially increased and subsequently decreased anisotropy in the MPO regions does reflect ongoing axonal regrowth, the exact relationship to the patient's clinical course is unknown. Since earlier studies are not available, we cannot rule out that the novel MPO feature may have existed prior to injury. The observation of a similar pattern of interval increase in anisotropy in the midline cerebellum, however, strongly supports the possibility of a slow recovery of intraregional connectivity through axonal remodeling, as this finding has a direct clinical correlate. Taken together, both observations in patient 1 suggest that axonal regrowth may play some role in very late recoveries of function in patients with severe brain injuries (17). The interval changes observed in the longitudinal study of patient 1 also provide insight into the possible connection of recovery of speech despite the lack of findings in language-related areas. If axonal reorganization is an ongoing 
process, these regions may have at one time experienced similar changes in connectivity not resolvable by DTI at this time but nonetheless consistent with the current observations. The suggestive findings of novel pathway development in the second patient cannot be further interpreted at this time.

Collectively, the findings support further efforts to prospectively and longitudinally characterize neuroplasticity in both brain structure and function following severe injuries (16). Studies of ongoing recovery utilizing DTI and PET beginning shortly after brain injury will also likely shed further light on debates surrounding the question of long-distance cortical rewiring (18) in humans and its potential importance in rehabilitation (19).

\section{Methods}

Our studies were approved by the Institutional Review Board (IRB) of Weill Medical College of Cornell University. IRB-approved consent declarations were obtained from all control subjects and the 2 patients' legally authorized surrogates.

MRI. Twenty normal control subjects (9 males, 11 females, age 19-54 years; median age, 25) and the 2 patients were scanned on the same GE Healthcare $3 T$ MRI Signa EXCITE scanner with 50 millitesla/m gradients, using a birdcage head coil in quadrature mode. The 20 control subjects were scanned within 9 weeks before and 5 weeks after the first scan of patient 1 . Patient 1 was again examined 18 months after the first scan. Patient 2 was scanned once only. An in-house 2D single-shot spinecho echo planar imaging (EPI) sequence with repetition time/echo time $(\mathrm{TR} / \mathrm{TE})=10000 / 100 \mathrm{~ms}$ was used for DTI. From 58 to 60 consecutive slices of 2.5 - $\mathrm{mm}$ thickness and axial matrix size of $128 \times 128$ were acquired in 1 number of acquisitions (NEX), with a field of view of $22 \mathrm{~cm}$. Additionally, T1-weighted anatomical images were acquired from all subjects. To test for reproducibility of the FA and diffusion constant histograms, another normal control subject was scanned twice within an interval of 17 months and 3 times within a week, showing good reproducibility (Figure 2, G and H).

$M R I$ data analysis. For quantification of right-left connectivity, we used FA and the right-left fiber directionality, $c$, which we defined as the absolute value of the $x$ component of the normalized principal eigenvector of the diffusion tensor. Both FA and $\chi$ range from 0 to 1 . These measures reflect the degree of anisotropy and the directionality of diffusion, respectively (20). FA and $\chi$ for the MPO region were computed as follows: the sagittal slice with the strongest intensity region of right-left fibers (appearing as red in FA color maps) in the MPO region was selected and a region of interest (ROI) of at least 75 voxels was drawn that encompassed the strongest FA values in that region. ROI-based quantification of anisotropy and diffusion always has some variability and may be biased depending on the subject performing the measurement. To reduce subjective bias, the reported FA and $\chi$ values for the MPO region of the first scan of patient 1 and the normal control subjects indicated in Figure 2J were averaged over 3 independent analyses by 3 of the authors. Furthermore, the anisotropy values for the vermis and MPO region of the second scan coincided with values obtained from a high-angular resolution diffusion imaging (HARDI) scan obtained the same day. For testing of significant differences between patient and con- trol values, we used a 1-tailed Wilcoxon rank sum test for both quantities. All other significances in differences in the mean of both quantities were evaluated by 2 -tailed Student's $t$ tests. For the second scan of patient 1 and for patient 2 , prior to computing quantitative values, the coordinate system of the head was aligned with the magnet coordinate system to adjust for necessary variations of head positioning for each patient. The diffusion gradient directions were rotated accordingly to ensure that in color anisotropy maps (21), the colors would correspond to the head coordinate system and the right-left directionality index to the true right-left direction in the head as well. To test for eddy current-induced distortions in the patients' images that may affect FA $(22,23)$, images of the quality of fit of the measured signal to the diffusion-attenuated signal, depending on the fitted diffusion tensor components, were used to assess the variability of the diffusion constant; these error plots showed no correlation with the MPO region or the vermis, and generally, the error in cortical areas was significant only for 1 to 2 voxels deep into the cortex. White matter fiber tracking was performed as described previously $(24,25,26)$.

PET-CT acquisition and analysis. Patient 1 was scanned twice, 1 day after the MRI scans in each case. FDG-PET was performed on a GE Healthcare combined PET-CT LS Discovery unit; $11.2 \mathrm{mCi}$ and $10.3 \mathrm{mCi}$ of FDG (at the first and second scan, respectively) were administered about $50 \mathrm{~min}$ utes before emission-mode PET scanning. A total of 35 transaxial slices of $4.25-\mathrm{mm}$ thickness with a matrix size of $128 \times 128$ and a field of view of $25 \mathrm{~cm}$ were acquired. The signal was acquired in dynamic high-sensitivity mode, yielding 210 images altogether, which were later averaged slicewise over time. For display (Figure 3), the resulting volumes were reinterpolated to sagittal slices of 4.88 -mm thickness, and the FDG uptake was color coded and overlaid to CT images prescribed collinear to the PET images. The unitless FDG standard uptake values, defined as regional activity per gram divided by injected dose per patient mass in grams, were measured in ROIs obtained from axial slices of the averaged images.

\section{Acknowledgments}

We thank the Charles A. Dana Foundation (N.D. Schiff) and the Weill Medical College General Clinical Research Center (M01 RR00047) for financial support of these studies. MRI scanning of control subjects was funded by the James S. McDonnell Foundation through a collaborative grant to the Brain Trauma Foundation. H.U. Voss acknowledges financial support from the Cervical Spine Research Society. Finally, we would like to thank the families of the 2 patients for their confidence in and support of this research.

Received for publication October 3, 2005, and accepted in revised form April 4, 2006.

Address correspondence to: Henning U. Voss, Citigroup Biomedical Imaging Center, Weill Medical College of Cornell University, 516 East 72nd Street, New York, New York 10021, USA. Phone: (212) 746-5781; Fax: (212) 746-6681; E-mail: hev2006@ med.cornell.edu.
1. Giacino, J.T., et al. 2002. The minimally conscious state - definition and diagnostic criteria. Neurology. 58:349-353.

2. Fins, J.J. 2003. Constructing an ethical stereotaxy for severe brain injury: balancing risks, benefits and access. Nat. Rev. Neurosci. 4:323-327.

3. Basser, P.J., Mattiello, J., and Le Bihan, D. 1994. Estimation of the effective self-diffusion tensor from the NMR spin echo. J. Magn. Res. B. 103:247-254.

4. Wakana, S., Jiang, H., Nagae-Poetscher, L.M., van Zijl,
P.C., and Mori, S. 2004. Fiber tract-based atlas of human white matter anatomy. Radiology. 230:77-87. 5. Liu, A.Y., Maldjian, J.A., Bagley, L.J., Sinson, G.P., and Grossman, R.I. 1999. Traumatic brain injury: diffusion-weighted MR imaging findings. AJNR Am. J. Neuroradiol. 20:1636-1641.

6. Carmichael, S.T., et al. 2005. Growth-associated gene expression after stroke: evidence for a growthpromoting region in peri-infarct cortex. Exp. Neurol. 193:291-311.
7. Napieralski, J.A., Butler, A.K., and Chesselet, M.F. 1996. Anatomical and functional evidence for lesion-specific sprouting of corticostriatal input in the adult rat. J. Comp. Neurol. 373:484-497.

8. Dancause, N., et al. 2005. Extensive cortical rewiring after brain injury. J. Neurosci. 25:10167-10179.

9. Bengtsson, S., et al. 2005. Extensive piano practicing has regionally specific effects on white matter development. Nat. Neurosci. 8:1148-1150.

10. McKinstry, M.C., et al. 2002. Radial organization of 
developing preterm human cerebral cortex revealed by non-invasive water diffusion anisotropy MRI. Cereb. Cortex. 12:1237-1243.

11. Mukherjee, P., and McKinstry, R.C. 2006. Diffusion tensor imaging and tractography of human brain development. Neuroimaging Clin. N. Am. 16:19-43.

12. Chklovskii, D.B., Mel, B.W., and Svoboda, K. 2004. Cortical rewiring and information storage. Nature. 431:782-788.

13. Pons, T.P., et al. 1991. Massive cortical reorganization after sensory deafferentation in adult macaques. Science. 252:1857-1860.

14. Peters, A., and Sethares, C. 2003. Is there remyelination during aging of the primate central nervous system? J. Comp. Neurol. 460:238-254.

15. Schiff, N., et al. 2002. Residual cerebral activity and behavioral fragments in the persistent vegetative state. Brain. 125:1210-1234.

16. Schiff, N.D., et al. 2005. fMRI reveals large-scale network activation in minimally conscious patients. Neurology. 64:514-523.

17. McMillan, T.M., and Herbert, C.M. 2004. Further recovery in a potential treatment withdrawal case 10 years after brain injury. Brain Inj. 18:935-940.

18. Pearson, P.P., Arnold, P.B., Oladehin, A., Li, C.X., and Waters, R.S. 2001. Large-scale cortical reorganization following forelimb deafferentation in rat does not involve plasticity of intracortical connections. Exp. Brain Res. 138:8-25.

19. Hallett, M. 2005. Neuroplasticity and rehabilitation. J. Rehabil. Res. Dev. 42:17-19.

20. Voss, H.U., et al. 2005. Regional increases in diffusion anisotropy after traumatic brain injury: a quantitative diffusion tensor imaging study. Proc. Intl. Soc. Mag. Reson. Med. 13:1368.

21. Pajevic, S., and Pierpaoli, C. 1999. Color schemes to represent the orientation of anisotropic tissues from diffusion tensor data: application to white matter fiber tract mapping in the human brain.
Magn. Reson. Med. 42:526-540.

22. Conturo, T.E., McKinstry, R.C., Aronovitz, J.A., and Neil, J.J. 1995. Diffusion MRI: precision, accuracy and flow effects. NMR Biomed. 8:307-332.

23. Basser, P.J, and Jones, D.K. 2002. Diffusion-tensor MRI: theory, experimental design and data analysis-a technical review. NMR Biomed. 15:456-467.

24. Watts, R., Liston, C., Niogi, S., and Ulug, A.M. 2003. Fiber tracking using magnetic resonance diffusion tensor imaging and its applications to human brain development. Ment. Retard. Dev. Disabil. Res. Rev. 9:168-177.

25. Voss, H.U., Watts, R., Ulug, A.M., and Ballon, D. 2006. Fiber tracking in the cervical spine and inferior brain regions with reversed gradient diffusion tensor imaging. Magn. Reson. Imaging. 24:231-239.

26. Voss, H.U., Watts, R., Borja, J., Ulug, A.M., and Ballon, D. 2005. Diffusion tensor imaging at $3 \mathrm{~T}$ with strongly reduced geometric and intensity distortions. Proc. Intl. Soc. Mag. Reson. Med. 13:11. 